\title{
LICHENS AND BRYOPHYTES OF THE TASMANIAN WORLD HERITAGE AREA I. MOUNT SPRENT
}

\author{
by G. Kantvilas and S.J. Jarman
}

(with two tables and an appendix)

Over 280 lichens and bryophytes were recorded during a botanical survey of Mount Sprent. The number of species is approximately twice that of the vascular species (136 species) and demonstrates the importance of lichens and bryophytes in assessing the botanical significance of the area. Six species, Catillaria contristans, Ochrolechia androgyna, Polychidium contortum, Thelotrema suecicum, Acromastigum verticale and Tylimanthus diversifolius are reported from Tasmania for the first time. Many of the species recorded are widespread in the high rainfall parts of Tasmania, but a significant number are confined mainly to the west. Lichen diversity is richest in subalpine and alpine heathland and on alpine rock outcrops, whilst bryophytes are most diverse in sheltered habitats such as young forest, scrub and amongst alpine rocks.

Key Words: Lichens, bryophytes, Tasmania.

In BANKS, M.R. et al. (Eds), 1991 (31:iii): ASPECTS OF TASMANIAN BOTANY - A TRIBUTE TO WINIFRED CURTIS. Roy. Soc. Tasm. Hobart: 149-162. https://doi.org/10.26749/rstpp.124.2.149

\section{INTRODUCTION}

The Tasmanian World Heritage Area was added to the UNESCO register of the world's natural and cultural heritage on 14 December 1982, in recognition of the region as the last great temperate wilderness in Australia and one of the last in the world. An area of 769360 ha was included and, in 1989, this was increased to approximately 1.37 million ha.

Major vegetation types represented in the World Heritage Area are cool temperate rainforest, buttongrass moorland, reeless alpine vegetation, wet eucalypt forest and scrub. In general, the vascular botany is well known (e.g. see Jarman \& Crowden 1978) and the concentration of Tasmanian endemics (Kirkpatrick \& Brown 1984) demonstrates the uniquely Tasmanian flavour of the region as a whole. In contrast, cryptogams as a group are very poorly documented and constitute a major and very obvious gap in the available biological data for the area. Despite recent advances in the study of the Tasmanian lichen flora (e.g. Kantvilas et al. 1985, Kantvilas \& Jarman 1988), little work has been conducted on a regional basis. With the exception of some very incomplete lists of lichens (Bratt 1976a, b, c, d, 1978) and mosses (Bratt 1978), there are no satisfactory inventories, either geographical or ecological, for any part of the area and no assessment of the regional peculiarities or conservation status of the flora.

This paper is the first detailed inventory of lichens and bryophytes for any part of the World Heritage Area. It deals with Mount Sprent, a major peak in the Wilmot Range in southwestern Tasmania. In terms of its vascular flora and geology, Mount Sprent can be considered typical of many mountains in southwestern Tasmania, and so provides some indication of the lichenological and bryological resources of the region.

\section{THE STUDY AREA}

Mount Sprent $\left(42^{\circ} 48^{\prime} \mathrm{S}, 145^{\circ} 57^{\prime} \mathrm{E}\right)$ rises $1059 \mathrm{~m}$ a.s.l. and lies at the northern end of the Wilmot Range, at the boundary of the Tasmanian World Heritage Area. To the east and northeast the mountain overlooks the hydroelectric impoundments of the Serpentine, Huon and Gordon Rivers whilst to the south, west and north it is bounded by wilderness. The mountain is composed of Precambrian metamorphic rocks, predominantly metaquartzite and pelitic sequences (Williams \& Corbett 1976). Its slopes support mainly buttongrass moorland, dominated by Gymnoschoenus sphaerocephalus, species of Restionaceae and shrubs of the Myrtaceae, Epacridaceae and Proteaceae. This forms a mosaic with scrub dominated by Banksia marginata and species of Leptospermum and Melaleuca. Small areas of wet sclerophyll forest and rainforest occur along river valleys, in protected gullies and on some of the lower slopes. Towards the summit, the vegetation grades into alpine heathland and lawn-like microshrubbery, sedgeland and herbfield. This vegetation also extends southwards along the range. The pinnacle consists mostly of sparsely vegetated, rocky crags. The nearest weather station, Strathgordon, about $8 \mathrm{~km}$ to the northeast (altitude $340 \mathrm{~m}$ ) has a mean annual rainfall of 
$2700 \mathrm{~mm}$, the wettest month being September $(325 \mathrm{~mm})$ and the driest January $(130 \mathrm{~mm})$ (Nunez 1979). Given its higher altitude, Mount Sprent can be expected to have a much higher rainfall. The coldest month at Strathgordon is July, with a mean maximum temperature of $9.2^{\circ} \mathrm{C}$, whilst the warmest month is January, with a mean maximum of $20.7^{\circ} \mathrm{C}$ (Nunez 1979). Snowfall is variable and may occur at any time of the year. Whilst there is little build-up of snow, some drifts may survive into summer.

\section{METHODS}

Sampling was undertaken on the northeastern face of the mountain from the beginning of the walking track at the Serpentine Dam (alt. $320 \mathrm{~m}$ ) to the summit $(1059 \mathrm{~m})$ and extended $1.5 \mathrm{~km}$ southward along the range down to an altitude of $860 \mathrm{~m}$. The work was carried out mainly on five days during January and February 1987, but data and collections from previous visits to the mountain were also incorporated into the results.

Habitats surveyed for lichens and bryophytes are described below. Each habitat was searched carefully and collections and records were made of each species encountered. An inventory of the vascular plants was compiled concurrently (appendix). Except for the young Leptospermum glaucescens forest (habitat type 1), several stands of each habitat were sampled. An abundance rating for lichens in each habitat was estimated subjectively in the field, but the same degree of detail was not possible for bryophytes which were recorded only as present or absent.

All lichens species were identified or checked in the laboratory using standard methods (e.g. White \& James 1985). Voucher specimens are held in the Tasmanian Herbarium (HO) with selected duplicates lodged in BG, BM, CBG, M, MB, NSW, NY, O and herb. Vezda. Species nomenclature follows Kantvilas (1989).

Bryophyte species were identified in the laboratory using light microscopy, and a number of specimens were sent to Australian or overseas specialists for examination. Voucher specimens are presently held in the personal herbarium of SJJ. Nomenclature generally follows Streimann \& Curnow (1989) for mosses and Ratkowsky (1987) for liverworts. The latter accords essentially with the classification of Scott \& Bradshaw (1986), except for the families Geocalycaceae and Schistochilaceae which follow Engel \& Schuster (1984) and Schuster \& Engel (1985) respectively.

\section{HABITATS}

(1) Young Leptospermum glaucescens forest Lowland. Vegetation dense, 6-8 $\mathrm{m}$ tall, dominated by Leptospermum glaucescens. Other species include Banksia marginata, Acacia mucronata, Phebalium squameum, Bauera rubioides, Monotoca submutica as well as occasional rainforest species such as Anodopetalum biglandulosum, Anopterus glandulosus, Cenarrhenes nitida, young Nothofagus cunninghamii, Trochocarpa cunninghamii and Blechnum wattsii.

(2) Buttongrass moorland

Lowland to subalpine. Low vegetation $(0.40 .8 \mathrm{~m}$ tall $)$ dominated by Gymnoschoenus sphaerocephalus, Leptospermum nitidum, Melaleuca squamea, Beackea leptocaulis, Sprengelia incarnata, Epacris corymbiflora and Restio monocephalus usually with emergent Banksia marginata 1-3 $\mathrm{m}$ tall. The peat surface is often visible in open ground between Gymnoschoenus tussocks.

(3) Nothofagus cunninghamii-Richea scoparia scrub Subalpine. Vegetation dense, $1-4 \mathrm{~m}$ tall, with an uneven canopy and gaps to ground level. Dominated by Nothofagus cunninghamii and Richea scoparia with Eucryphia milliganii sometimes common. Other widespread species present include Monotoca submutica, Richea milliganii, Olearia persoonioides, Helichrysum backhousil, Gahnia grandis, Melaleuca squamea, Bauera rubioides, Empodisma minus, Dracophyllum milliganii and Isophysis tasmanica.

(4) Alpine lawns and associated sedgelands

Alpine. Very dense vegetation $50-100 \mathrm{~mm}$ tall. Diversity is high and common species include Isophysis tasmanica, Xyris marginata, Oreobolus oligocephalus, Donatia novae-zelandiae, Empodisma minus and Calorophus sp.nov. In appearance, the community is like a compact lawn but its surface becomes more uneven as Dracophyllum milliganii or Carpha curvata become more abundant.

(5) Sheltered rock sites (bryophyte habitat)

Subalpine to alpine. This habitat occurs in most vegetation types where rocks are large enough or occur together so that cool, shady and moist microhabitats are maintained at ground level. The rocks are often surrounded by low thickets of shrubs which help preserve the cool, humid environment.

(6) Exposed rock sites (bryophyte habitat)

Lowland to alpine. A harsh environment where the rock surface protrudes above the surrounding vegetation and receives little protection from wind, heat or sunlight. 
(7) Low altitude rock outcrops (lichen habitat)

Large outcrops of bedrock are scattered through the buttongrass moorland, frequently surrounded by scrubby vegetation containing additional shrubs such as Cenarrhenes nitida and Monotoca submutica. Lichens occur in peat-filled crevices or directly on rock. Similar to the exposed rock site habitat, listed above, but restricted to low altitudes.

(8) Alpine rocks (lichen habitat)

(9) Alpine heathland (lichen habitat)

Low shrubby vegetation dominated by Eucalyptus vernicosa, Monotoca submutica, Nothofagus cunninghamii, Epacris serpyllifolia, Cenarrhenes nitida and Persoonia gunnii. It rarely forms extensive continuous tracts but occurs as small patches between rock outcrops and crags.

\section{RESULTS}

\section{Lichens}

For lichens, 141 taxa in 66 genera were recorded (table 1), including 95 macrolichens and 46 crustose species. Major genera with respect to prominence and diversity among the macrolichens were Cladia ( 7 taxa), Cladonia (14), Menegazzia (5), Pseudocyphellaria (8), Psoroma (6), Siphula (6), Sphaerophorus (6) and Usnea (5). Amongst the crustose lichens, the genera of the Lecideaceae s.lat. (Bacidia, Catillaria, Fuscidea, Lecidea, Lecidella, Mycoblastus, Paraporpidia, Poeltiaria and Tremolecia) and those of the Pertusariaceae (Coccotrema, Melanaria, Ochrolechia and Pertusaria) tended to be most abundant.

Four species were recorded for Tasmania for the first time: Catillaria contristans, Ochrolechia androgyna and Thelotrema suecicum are cool temperate Northern Hemisphere species, whilst Polychidium contortum was previously considered endemic to New Zealand (Galloway 1985). However, several other species, reported elsewhere in the literature (e.g. Kantvilas 1989), were also first discovered in Tasmania during this present survey.

The endemic Tasmanian lichens found during the study include Cladia moniliformis, Menegazzia subbullata, Sarrameana tasmanica and Siphula jamesii. Several of the undescribed species (e.g. in the genera Baeomyces s.lat,, Melanaria, Ramonia and Siphula) are currently the focus of further study and are likely to be endemic to Tasmania as well. Notes on these taxa are given as a footnote to table 1.

Approximately 43 species are terricolous, 52 are saxicolous or occur amongst bryophytes over rocks, and 73 are epiphytic. However, some species, such as Siphula decumbens and Cladia aggregata, span several habitats. Indeed a general characteristic of Precambrian rock outcrops in southwestern Tasmania is that numerous species which are typically epiphytic occur in moist, sheltered crevices, e.g. Megalospora lopadioides and Sphaerophorus spp. The most diverse habitat types on Mount Sprent are subalpine and alpine heathland and alpine rock outcrops.

\section{Bryophytes}

For bryophytes, 146 species were recorded (table 2, pp.156-159), including 48 mosses and 98 liverworts. Excluding two unidentified species, mosses are represented by 34 genera in 23 families (classification after Scott \& Stone 1976). The family which is best represented is the Dicranaceae (10 species) and it contains the two most widespread and abundant mosses recorded, Campylopus introflexus and Dicranoloma robustum. The liverworts comprise 86 leafy and 12 thalloid species and, with five unidentified species excluded, are divided into 43 genera and 21 families. The Geocalycaceae, represented by common genera such as Heteroscyphus and Chiloscyphus, and the Lepidoziaceae, which includes genera such as Acromastigum, Lepidozia and Telaranea, are the largest families present with 19 and 18 species respectively.

Acromastigum verticale, previously known only from New Zealand, was recorded from Tasmania for the first time, and the presence of Tylimanthus diversifolius, previously listed as doubtful for Tasmania (Scott 1985) was confirmed. A rare species, Pulchrinodus inflatus, which we discovered only recently in Tasmania from the De Witt and Propsting Ranges further to the southwest (Allen 1987), was also recorded.

Six endemic species were recorded. These include three mosses (Dicranoweissia microcarpa, Tayloria gunnii and $T$. tasmanica) and three liverworts (Brevianthus flavus from the monotypic family Brevianthaceae, Schistochila tasmanica and Herbertus oldfieldianus).

The majority of bryophytes were soil or rock colonising species. A few obligate epiphytes were present but most epiphytic species were found on stems near ground level and occurred also on soil, dead wood or rocks. The highest number of species was found in young forest, scrub and amongst sheltered alpine rocks, all of which contain the greatest diversity of microhabitats. Exposed habitats such as alpine "lawns" and buttongrass moorland were relatively impoverished, although species richness increased where rocks or clumps of emergent shrubs provided moist, sheltered microhabitats. 
TABLE 1

Lichens Recorded from Mount Sprent, Showing Qualitative Abundance* in Seven Habitat Types $\uparrow$

Austroblastenia pauciseptata (Shirley) Sipman

A. pupa Sipman

0

Bacidia sp. A ${ }^{1}$

Baeomyces heteromorphus Nyl. ex Church.

Bab. \& Mitten

Baeomyces s.lat. sp.nov. ${ }^{2}$

Catillaria contristans (Nyl.) Zahlbr.

Catillaria sp. $\mathrm{A}^{3}$

Chiodecton cf. colensoi (Massal.) Müll. Arg.

Cladia aggregata (Sw.) Nyl.

C. fuliginosa Filson

C. inflata (F.Wilson) D.Galloway

C. moniliformis Kantvilas \& Elix

C. retipora (Labill.) Nyl.

C. schizopora (Nyl.) Nyl.

C. sullivanii (Müll.Arg.) W.Martin

Cladina confusa (R.Sant.) Follm. \& Ahti

C. mitis (Sandst.) Hustich

Cladonia capitellata (J.D.Hook. \& Taylor)

Church. Bab. var. capitellata

C. capitellata var. interhiascens ( $\mathrm{Nyl}$.)

Sandst.

C. cervicornis (Ach.) Flotow ssp. verticillata

(Hoffm.) Ahti

C. cornuta (L.) Hoffm.

C. crispata (Ach.) Flotow 4

C. gracilis (L.) Willd. ssp. tenerrima Ahti

C. kuringaiensis Archer

C. murrayi W.Martin

C. pleurota (Flörke) Schaerer

C. ramulosa (With.) Laundon

C. rigida (Taylor) Hampe var. rigida

C. southlandica W.Martin

C. subdigitata Nyl.

C. weymouthii F.Wilson ex Archer

Coccotrema cucurbitula (Mont.) Müll.Arg.

C. porinopsis (Nyl.) Imshaug ex Yoshimura

Coccotrema sp. 5

Degelia durietzii Arvidsson \& D.Galloway

Dendriscocaulon dendriothamnodes Dughi

Habitat

$1 \quad 2 \quad 3$

4

7

8

9

o

(n)

o

o

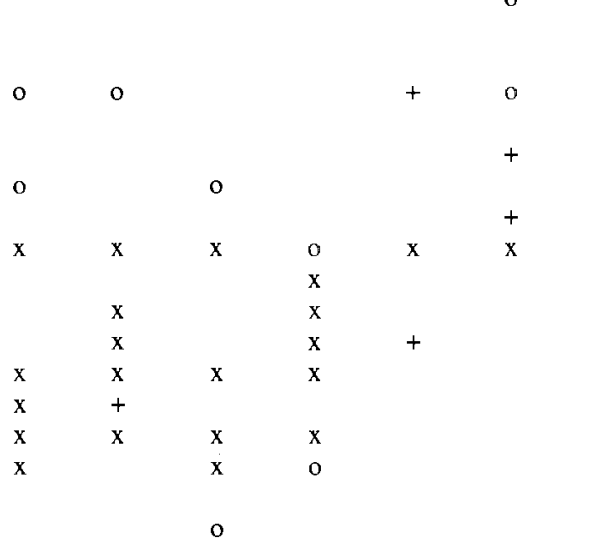

o

$+$

o

X

$\mathrm{X}$

$\mathrm{x}$

$\mathrm{X}$

$\mathrm{X}$

$\mathrm{x}$

o

$$
\text { o }
$$

$$
\mathrm{x}
$$$$
\text { o }
$$ 


\begin{tabular}{|c|c|c|c|c|c|c|c|}
\hline & & & & Habita & & & \\
\hline & 1 & 2 & 3 & 4 & 7 & 8 & 9 \\
\hline Graphis insidiosa (Knight \& Mitten) J.D.Hook. & + & & & & & & \\
\hline Hypogymnia lugubris (Pers.) Krog & & o & o & & & $\mathrm{x}$ & $\mathrm{x}$ \\
\hline H. turgidula (Bitter) Elix s.lat. & 0 & o & & & & & \\
\hline Hypotrachyna sinuosa $(\mathrm{Sm}$.) Hale & & + & & & + & & \\
\hline $\begin{array}{l}\text { Knightiella splachnirima (J.D.Hook. \& } \\
\text { Taylor) Gyelnik }\end{array}$ & $o$ & & o & & & & $\mathrm{x}$ \\
\hline Lecanora blanda Nyl. & & & & & o & o & \\
\hline Lecanora $\mathrm{sp}^{6}$ & & & & & & + & \\
\hline Lecidea sp. $^{7}$ & & & & & & & + \\
\hline L. lapicida (Ach.) Ach. & & & & & & 0 & \\
\hline Lecidella elaeochroma (Ach.) Choisy & & o & $\mathrm{x}$ & & & & $\mathrm{x}$ \\
\hline Leioderma pycnophorum Nyl. & & & $\mathrm{x}$ & & & & + \\
\hline Lepraria incana (L.) Ach. aggr. & & & + & & & & + \\
\hline Megalospora lopadioides Sipman & + & & 0 & & & o & $\mathrm{x}$ \\
\hline Melanaria sp. 8 & & & & $\mathrm{x}$ & & + & 0 \\
\hline Menegazzia aeneofusca (Müll.Arg.) R.Sant. & & & & & & + & \\
\hline M. globulifera R.Sant. & & & & & & & + \\
\hline M. subbullata P.James \& Kantvilas & + & + & & & & & \\
\hline M. testacea P.James \& D.Galloway & & & & & & & + \\
\hline M. weindorferi (Zahlbr.) R.Sant. & & + & + & & & & \\
\hline Micarea austroternaria Coppins \& Kantvilas & & $\mathrm{x}$ & & o & & o & $\mathbf{x}$ \\
\hline M. flagellispora Coppins \& Kantvilas & & & o & & & & $\mathrm{x}$ \\
\hline M. isabellina Coppins \& Kantvilas & & $\circ$ & & o & & & \\
\hline Multiclavula vernalis (Schw.) Petersen & & & & o & & & \\
\hline Mycoblastus sp.l 9 & + & & o & & & & o \\
\hline Mycoblastus sp.2 & $\mathrm{x}$ & $\mathrm{x}$ & & & & & \\
\hline Mycoblastus sp. 3 & $\mathrm{x}$ & $\mathrm{x}$ & & & & & $\mathrm{x}$ \\
\hline Neofuscelia stygiodes (Nyl. ex Crombie) Esslinger & & & & & o & $\mathrm{x}$ & \\
\hline Neophyllis melacarpa (F.Wilson) F.Wilson & & & & & & 0 & o \\
\hline Nephroma sp. 10 & & & & & & & + \\
\hline Ochrolechia androgyna (Hoffm.) Arn. & & & & & & & + \\
\hline Omphalina sp. & & & & & & & + \\
\hline $\begin{array}{l}\text { Paraporpidia leptocarpa (Church. Bab. \& Mitten) } \\
\text { Rambold \& Hertel }\end{array}$ & & & & & $\mathrm{x}$ & $\mathrm{x}$ & \\
\hline Parmelia signifera Nyl. & & & & & o & $\mathrm{x}$ & \\
\hline Parmeliella nigrocincta (Mont.) Müll.Arg. aggr. & & & + & & & & \\
\hline Parmelina stevensiana Elix \& Johnston & & + & & & & & \\
\hline Peltigera dolichorhiza (Nyl.) Nyl. & & & + & & & & \\
\hline Pertusaria gymnospora Kantvilas & & & & & & & $\mathbf{x}$ \\
\hline P. superba Zahlbr. & & & & & o & $\mathrm{x}$ & \\
\hline P. truncata Krempelh. & + & & $\mathrm{x}$ & & & & 0 \\
\hline $\begin{array}{l}\text { Phaeographis exaltata (Mont. \& v.d. Bosch) } \\
\text { Müll.Arg. }\end{array}$ & + & & & & & & \\
\hline $\begin{array}{l}\text { Poeltiaria coromandelica (Zahlbr.) Rambold } \\
\quad \text { \& Hertel }\end{array}$ & & & & & o & $\mathrm{x}$ & \\
\hline Polychidium contortum Henssen & & & $\mathrm{x}$ & & & & 0 \\
\hline Porina sp. ${ }^{11}$ & & & + & & & & \\
\hline P. leptaleina (Nyl.) Müll.Arg. & & & + & & & & \\
\hline Protoparmelia petraeoides (Nyl. ex Hue) Hertel & & & & & & $o$ & \\
\hline Pseudocyphellaria billardierei (Delise) Räsänen & & & 0 & & & & \\
\hline P. colensoi (Church.Bab.) Vainio & & & + & & & & \\
\hline
\end{tabular}




P. coronata (Mïll.Arg.) Malme
P. faveolata (Delise) Malme
P. glabra (J.D.Hook. \& Taylor) Dodge
P. insculpta (Sizenb.) D. Galloway
P. multifida (Nyl.) D.Galloway \& P.James
P. rubella (J.DHook. \& Taylor) D.Galloway
$\quad$ \& P.James
Psora sp. 12
Psoroma asperellum Nyl.
P. durietzii P.James \& Henssen
P. leprolomum (Nyl.) Räsänen
P. microphyllizins (Nyl.) D.Galloway
P. pholidotoides (Nyl.) Trevisan
P. soccatum R.Br.
Psoromidium versicolor (J.D.Hook. \& Taylor)
$\quad$ D.Galloway
Pycnothelia caliginosa D.Galloway \& P.James

Ramonia s.1at. sp.nov. ${ }^{13}$

Rhizocarpon cf. geographicum (L.) DC.

Habitat

$R$. cf. grande (Flörke) Arnold

Sarrameana tasmanica Vezda \& Kantvilas

Siphula complanata (J.D.Hook. \& Taylor)

R.Sant. aggr.

S. decumbens Nyl.

S. fragilis (J.D.Hook. \& Taylor) J.Murray

S. jamesii Kantvilas

$S$. aff. ramalinoides Nyl. ex Crombie

Siphula sp.nov.

Siphulastrum mamillatum (J.D.Hook, \&

Taylor) D.Galloway

Sphaerophorusinsignis Laurer

S. macrocarpus Ohlsson in D.Galloway

S. melanocarpus (Sw.) DC.

S. ramulifer Lamb

S. scrobiculatus (Church.Bab.) Sato

S. tener Laurer

Stephanocyclos henssenianus Hertel

Stereocaulon caespitosum Redinger

S. corticatulum Nyl.

S. gregarium Redinger

S. ramulosum (Sw.) Räuschel

Sticta limbata (Sm.) Ach.

S. stipitata Knight

S. sublimbata (Steiner) Swinscow \& Krog

o

$\mathrm{X}$

o

0

0

o

o

$+$

0

o

o

o

o

0

$\mathrm{X}$

$x \quad x$

o

$+$

$\mathrm{X}$

$\mathrm{X}$

o

$\mathrm{x}$

$\mathrm{x}$

$\mathrm{X}$

$\mathrm{x}$

$\mathrm{x}$

$\mathrm{x}$

o

o

o

o

o

$+$

o

0

$+$

$\mathrm{x}$

o

o

+
$+\quad+$
$\mathrm{x}$
$\mathrm{o}$

Tephromela atra (Huds.) Hafellner

Thelotrema decorticans Müll.Arg.

T. suecicum (H.Magn.) P.James

Thelotrema s.lat. sp.nov. 14

Tremolecia atrata (Ach.) Hertel

o

$\mathrm{x}$

Umbilicaria cylindrica (L.) Delise in Duby

U.polyphylla (L.) Baumg.

Usnea capillacea Motyka

U. molliuscula Stirton

U. torulosa (Müll.Arg.) Zahlbr.

0

o 


\begin{tabular}{|c|c|c|c|c|c|c|c|}
\hline & \multicolumn{7}{|c|}{ Habitat } \\
\hline & 1 & 2 & 3 & 4 & 7 & 8 & 9 \\
\hline U. xanthopoga Nyl. & & o & $t$ & & & & \\
\hline Wawea fruticulosa Henssenn \& Kantvilas & & & $\mathrm{O}$ & & & & \\
\hline $\begin{array}{l}\text { Xanthoparmelia mougeotina (Nyl.) } \\
\text { D. Galloway }\end{array}$ & & & & & $x$ & $\mathrm{x}$ & \\
\hline X. xanthomelaena (Müll.Arg.) Nash \& Elix & & & & & & o & \\
\hline Total number of taxa & 35 & 24 & 54 & 19 & 25 & 53 & 60 \\
\hline
\end{tabular}

* $\mathrm{x}=$ common, $\mathrm{o}=$ occasional, $+=$ rare.

$\dagger$ See text under "Habitats".

1 Saxicolous species with black apothecia, dark-brown epithecium, colourless hymenium and hypothecium, and narrow

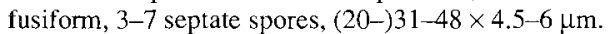

2 New monotypic genus widespread in southwestem Tasmania (G.Kantvilas, P.W. James \& J.A. Elix, unpubl.).

3 Common and widespread corticolous species characterised by milky white to pale fawn-pink apothecia, pale-brown, K+ yellowish epithecium, colourless hymenium and hypothecium, and, 1 -septate, ellipsoid spores, 9.5 $15(-18) \times 4-5(-10) \mu \mathrm{m}$.

4 Chemical strain containing thamnolic acid, with barbatic acid in the apothecia.

5 Undescribed, coarsely sorediate species containing atranorin only.

6 Saxicolous species related to Lecanora cenisea Ach. from the Northem Hemisphere (P.James, pers. comm.).

7 Uncommon corticolous species with very thin, effuse pale green-grey thallus, black, plane apothecia with a thin, persistent margin and ellipsoid spores, 15.5-19.5 × 12-15.5 $\mu \mathrm{m}$.

8 Widespread and common on Tasmanian mountains; characterised by a thick, pale-yellow thallus which encrusts peat, litter and microshrubbery; see Kantvilas \& Jarman (1988) under Pertusaria.

9 Corticolous species with black, immarginate apothecia and large, simple spores (1-)2(-3) per ascus, 38-72 $\times 20-36 \mu \mathrm{m}$. The three taxa listed differ in the internal coloration of their apothecia: sp. 1 - epithecium brown to blue-green, $\mathrm{K}+$ greenish grey, hymenium pale brown, colourless in $\mathrm{K}$, and hypothecium pale brown, colourless or yellowish in $\mathrm{K}$; sp.2 - epithecium purple, $\mathrm{K}+$ blue-green, hymenium and hypothecium pale brown, colourless in $\mathrm{K}$, and subhypothecium $\mathrm{K}+$ golden yellow; sp. 3 epithecium blue-green, $\mathrm{K}+$ yellow, and remainder of apothecium pale brown, $\mathrm{K}+$ blood red.

10Rare corticolous species with cyanobacteria, containing methyl gyrophorate (P.M. Jørgensen, pers. comm.).

11 Corticolous, with black perithecia and 5-7 septate spores, $30-52 \times 2 \mu \mathrm{m}$ with acute apices and frequently twisted once in the centre.

12Common, undescribed, terricolous, alpine species, probably referable to a new genus (E. Timdal, pers. comm.).

13Undescribed species related to Ramonia stipitata Vezda \& Hertel from Venezuela. Common on Richea scoparia at high altitudes in Tasmania.

14Common, widespread, corticolous or muscicolous species with large apothecia with thick exfoliating margins and muriform spores, eight per ascus, $45-70 \times 10-19 \mu \mathrm{m}$. 


\begin{tabular}{llllllll}
\hline Habitat & & & & & \\
& 1 & 2 & 3 & 4 & 5 & 6
\end{tabular}

\section{Mosses}

Achrophyllum dentatum (J.D.Hook. \& Wils.) Vitt \& Crosby Andreaea acutifolia J.D.Hook. \& Wils.

$\mathrm{x}$

A. mutabilis J.D.Hook. \& Wils.

Bartramiaithyphylla Brid.

Breutelia pendula (Smith) Mitt.

Bryum sp.

Campylopus clavatus (R.Br.) Wils.

C. introflexus (Hedw.) Brid.

C. kirkii Mitt.

Conostomum pusillum J.D.Hook. \& Wils.

Cyathophorum bulbosum (Hedw.) C.Muell.

Dicranoloma billardieri (Brid. ex anon.) Par.

D. dicarpum (Nees.) Par.

D. eucamptodontoides (Broth. \& Geh.) Par.

D. menziesii (Taylor) Par.

D. robustum (J.D. Hook. \& Wils.) Par.

D. trichopodum (Mitt.) Broth.

Dicranoweissia microcarpa (J.D.Hook. \& Wils.) Par.

Distichophyllum pulchellum (Hampe) Mitt.

Ditrichum punctulatum Mitt.

Hypnodendron comosum (Labill.) Mitt.

Hypnum cupressiforme Hedw.

Leptostomum inclinans $\mathrm{R} . \mathrm{Br}$.

Leptotheca gaudichaudii Schwaegr.

Leucobryum candidum (Beauv.) Wils.

Macromitrium archeri Mitt.

Plagiothecium denticulatum (Hedw.) B.S.G.

Pleurophascum grandiglobum Lindb.

Polytrichum juniperinum Hedw.

P. formosum Hedw.

Psilopilum crispulum (J.D.Hook. \& Wils.) Mitt.

Ptychomnion aciculare (Brid.) Mitt.

Pulchrinodus inflatus (J.D.Hook. \& Wils.) Allen

$\mathrm{x}$

$\mathrm{X}$

$\mathrm{X}$

$\mathrm{X}$ $\mathrm{x}$

$\mathrm{X}$

$\mathrm{x} \quad \mathrm{x}$

$\mathrm{x}$

$\mathrm{x}$

$\mathrm{X}$

$\mathrm{X}$

$X$

$\mathrm{X}$

$\mathrm{x}$

$\mathbf{x}$

$x$

$\mathrm{X}$

$\mathrm{x}$

$\mathrm{X}$

$\mathrm{X}$

$\mathbf{x}$

$\mathrm{X}$

$x$

Racomitrium crispulum (J.D.Hook. \& Wils.) J.D.Hook. \& Wils.

R. lanuginosum (Hedw.) Brid.

Rhacocarpus purpurascens (Brid.) Par.

Rhaphidorrhynchium amoenum (Hedw.) Fleisch.

Rhizogonium pennatum J.D.Hook. \& Wils.

$\begin{array}{ll} & \mathrm{x} \\ \mathrm{X} & \mathrm{X} \\ \mathrm{X} & \mathrm{X}\end{array}$

$\mathrm{X}$

$\mathrm{x}$

$x$

$\mathrm{X}$

$\mathrm{X}$

Sematophyllum sp.

Sphagnum australe Mitt. 


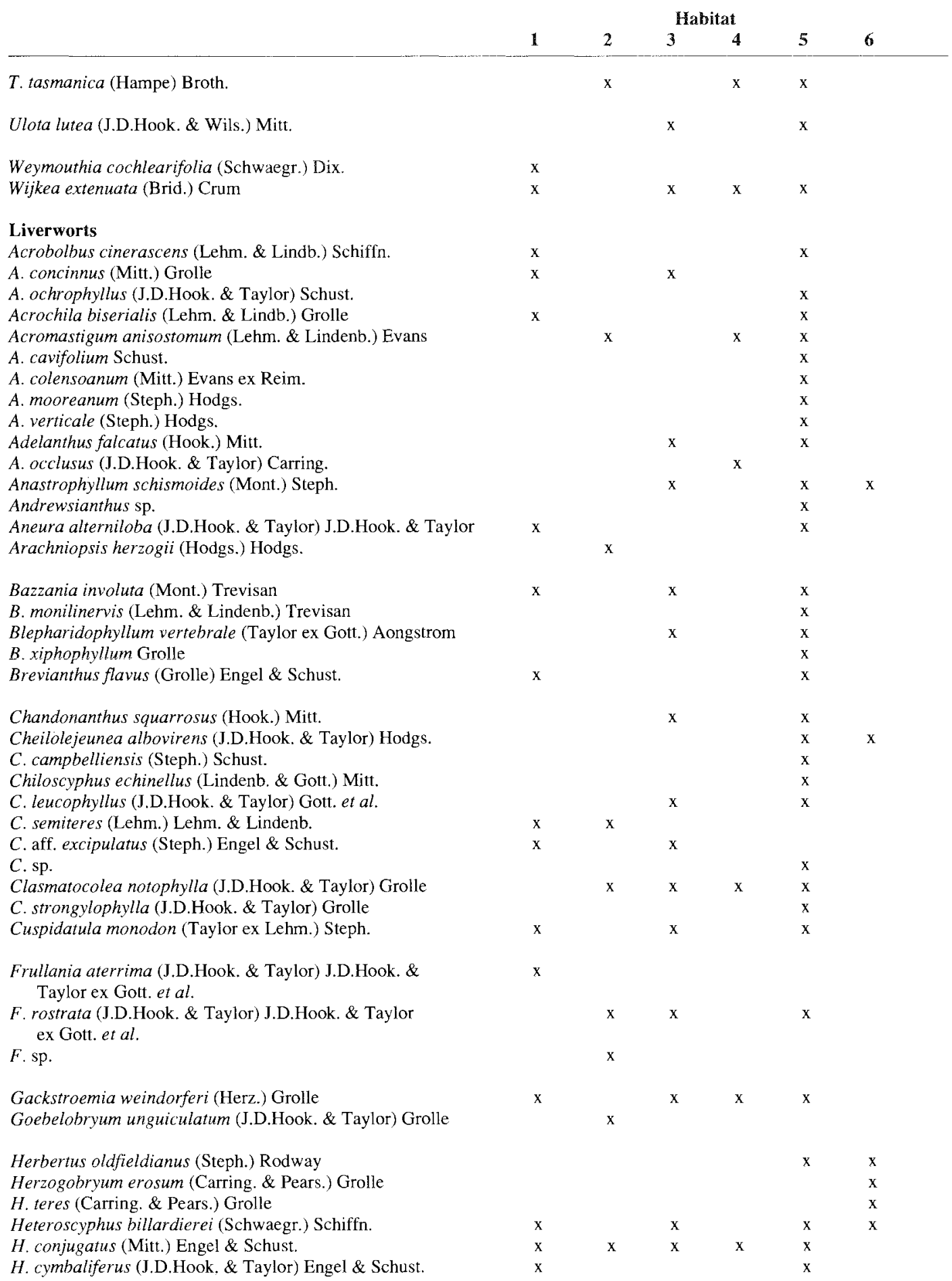




H. decipiens (Gott.) Engel \& Schust.
H. fissistipus (J.D.Hook. \& Taylor) Schiffn.
H. levieri (Steph.) Schiffn.
H. tasmanicus (Steph.) Engel \& Schust.
H. weymouthianus (Steph.) Schiffn.

H. sp.

Isolembidium anomalum (Rodway) Grolle

Isotachis intortifolia (J.D.Hook. \& Taylor) Gott. et al.

Jamesoniella colorata (Lehm.) Spruce ex Schiffn.

Kurzia sp.

Lepicolea scolopendra (Hook.) Dum. ex Trevisan

Lepidozia glaucophylla (J.D.Hook. \& Taylor) Taylor ex Gott. et al.

L. ulothrix (Schwaegr.) Lindenb.

Leptophyllopsis laxa (Mitt.) Schust.

Leptoscyphus sp.

Marsupidium surculosum (Nees) Schiffn.

Metzgeria saccata Mittn.

M. aff. decipiens (Massal.) Schiffn. \& Gott.

$M$. aff. furcata (L.) Dum.

$M$. sp.

Paraschistochila pinnatifolia (Hook.) Schust.

P. tuloides (J.D.Hook. \& Taylor) Schust.

Plagiochila circinalis (Lehm.) Lehm. \& Lindenb.

$P$. fasciculata Lindenb.

$P$. radiculosa Mitt.

$P$. retrospectans Nees

P. strombifolia (Taylor) Taylor ex Lehm.

Podomitrium phyllanthus (Hook.) Mitt.

Radula compacta Castle

R. multiamentula Hodgs.

$R$. ratkowskiana Yamada

$R$. tasmanica Steph.

Riccardia aequicellularis (Steph.) Hewson

$R$. cochleata (J.D.Hook. \& Taylor) Kuntze

$R$. crassa (Schwaegr.) Carring. \& Pears.

$R$. aff. bipinnatifida (Col.) Hewson

$R . \mathrm{sp}$.

Saccogynidium decurvum (Mitt.) Grolle

Schistochila lehmanniana (Lindenb.) Carring. \& Pears. 


\section{Habitat}

$\begin{array}{llllll}1 & 2 & 3 & 4 & 5 & 6\end{array}$

T. tenellus (J.D.Hook. \& Taylor) Hodgs.

Zoopsis argentea (J.D.Hook. \& Taylor) J.D.Hook. ex Gott. et $\boldsymbol{a l}$.

Z. leitgebiana (Carring. \& Pears.) Bastow

Z. setulosa Leitg.

Total number of taxa

* See text under Habitats.

\section{DISCUSSION}

Lichens and bryophytes constitute a significant component of the botanical resources of Mount Sprent, with over 280 species being recorded. This number could be expected to increase significantly if the survey were extended to include rainforest, one of the richest habitats in Tasmania for cryptogams but not easily accessible on the mountain. Comparing vegetation from the same sites, lichens and bryophytes outnumber the vascular species by at least two to one, the latter being represented by 121 angiosperms, two gymnosperms and 13 pteridophytes (appendix 2). These figures demonstrate unequivocally the importance of nonvascular plants in any sensible assessment of the conservation values and management of the region. However, the results should not be extrapolated to all of southwestern Tasmania or all of the World Heritage Area as these areas include a wide range of unsampled habitats which, for the most part, remain cryptogamically unexplored.

The high rate of endemism amongst the vascular plants ( $63 \%$ for the angiosperms) is not matched by the lichens and bryophytes, where only approximately $3 \%$ of the bryophytes and 5\% of the lichens are endemic to Tasmania. However, these low figures are consistent with the low rates of endemism these groups display in other regions and vegetation types in Tasmania (Kantvilas et al. 1985, Kantvilas \& Jarman 1988, Ratkowsky \& Ratkowsky 1982).

The majority of lichens recorded, including the most common species, are widely distributed at least in the wetter, western parts of Tasmania. Thus the flora of Mount Sprent includes 35\% of the lichens recorded from rainforest (Kantvilas 1985, Kantvilas \& James 1987 ) and $60 \%$ of those from buttongrass moorland (Kantvilas \& Jarman 1988). A significant group of species is distinctly western in its Tasmanian distribution including Baeomyces s.lat. sp.nov., Cladia moniliformis, Fuscidea absolodes, Micarea isabellina, Multiclavula vernalis, Pertusaria gymnospora, Pycnothelia caliginosa, Siphula sp.nov., S. jamesii and Stephanocyclos henssenianus. In the absence of published ecological information, similar comparisons are not yet possible for the bryophytes. However, approximately one-third of the species from Mount Sprent are also known from the Wellington Range in southeastern Tasmania (Ratkowsky \& Ratkowsky 1982).

Mount Sprent is noticeably depauperate with respect to some common lichen genera, particularly Xanthoparmelia (which typically abounds in areas where bare rock surfaces prevail) and Menegazzia (only five species present out of a possible 17). There is also an apparent paucity of high alpine lichens: genera such as Placopsis, Coelocaulon, Alectoria, Cetraria, Pseudephebe, Thamnolia and Arthroraphis are absent, as are certain characteristic alpine species of Umbilicaria, Usnea, Psoroma and Hypogymnia. The only truly alpine Tasmanian lichens present are Stereocaulon caespitosum (which is rare), Cladia fuliginosa and Cladina mitis. This may be due to the relatively low altitude of the peak, or it may be related to geology, given that most of the genera listed above are most common on dolerite peaks. Rock type would not be expected to influence the occurrence of epiphytic species or of those growing on peat, and the distribution of these lichens may have been influenced by fire history. Mount Sprent forms only a small alpine pinnacle in a landscape of buttongrass moorland and may have been burnt frequently in the past. The absence of any extensive alpine environments nearby could mean that once certain restricted, high altitude species have been eliminated, their opportunities for recolonisation from unburnt alpine refugia become minimal. 


\section{ACKNOWLEDGEMENTS}

We thank A.W. Archer, B.J. Coppins, J.A. Elix, J.J. Engel, R. Grolle, H. Hertel, P.W. James, P.M. Jørgensen, D.I. Morris, D.A. Ratkowsky, G.A.M. Scott, H.J.M. Sipman, E. Timdal and A. Vezda for comments on or identification of selected taxa. The study was funded by a World Heritage Area wildlife research grant.

\section{REFERENCES}

Allen, B.H., 1987: A systematic account of Pulchrinodus inflatus (Musci: Pterobryaceae), genus novum. NZ J. Bot. 25: 335-342.

BRatT, G.C., 1976a: Lichens of South West Tasmania. I. Lichens of the buttongrass areas. Tasm. Nat. 45: $1-4$.

Bratr, G.C., 1976b: Lichens of South West Tasmania. II. Mountain peaks and plateaux. Tasm. Nat. 46: 14.

BRatT, G.C., 1976c: Lichens of South West Tasmania. III. Forests. Tasm. Nat. 47: 1-4.

Bratt, G.C., 1976d: Lichens of the Cradle Mountain area. Tasm. Tramp 22: 111-118.

BRatT, G.C., 1978: Mosses and lichens. In Sharp-Paul, A. (Ed.): LOWER GORDON RIVER SCIENTIFIC SURVEY. Hydro-Electric Commission, Hobart: $1-24$.

Buchanan, A.M., McGeary-Brown, A. \& Orchard, A.E., 1989: A CENSUS OF THE VASCULAR PLANTS OF TASMANIA. Tasm. Herb. Occ. Publ. 2: 1-82.

Engel, J.J. \& SChuster, R.M., 1984: An overview and evaluation of the genera of Geocalycaceae subfamily Lophocoleoideae (Hepaticae). Nova Hedwigia 39: $385-463$

Galloway, D.J., 1985: FLORA OF NEW ZEALAND LICHENS. Govemment Printer, Wellington.

JARMAN, S.J. \& CROWDEN, R.K., 1978: A survey of vegetation. In Sharp-Paul, A. (Ed.): LOWER GORDON RIVER SCIENTIFIC SURVEY. Hydro-Electric Commission, Hobart.

Kantvilas, G., 1985: Studies on Tasmanian Rainforest Lichens. Unpubl. PhD thesis, University of Tasmania.

Kantvilas, G., 1989: A checklist of Tasmanian lichens. Pap. Proc. R. Soc. Tasm. 123: 67-85.

Kantvilas, G. \& JAMES, P.W., 1987: The macrolichens of Tasmanian rainforest: key and notes. Lichenologist 19: $1-28$.
Kantvilas, G., James, P.W. \& Jarman, S.J., 1985 Macrolichens in Tasmanian rainforests. Lichenologist 17: 67-83.

Kantvilas, G. \& JARMAN, S.J., 1988: Lichens of buttongrass (Gymnoschoenus) moorland in Tasmania. Pap. Proc R. Soc. Tasm. 122: 1-17.

Kirkpatrick, J.B. \& Brown, M.J., 1984: A numerical analysis of Tasmanian higher plant endemism. Bot. J. Linn. Soc. 88: 165-183.

NunEz, M., 1979: Climate. In Gee, H. \& Fenton, J. (Eds): THE SOUTH WEST BOOK. Australian Conservation Foundation, Melbourne: 67-69.

RatKowSKY, D.A., 1987: Checklist of Tasmanian liverworts. Pap. Proc. R. Soc. Tasm. 121: 153-158.

Ratkowsky, D.A. \& Ratkowsky, A.V., 1982: The bryophytes of the Mt Wellington Range, Tasmania. Pap. Proc. R. Soc. Tasm. 116: 97-115.

SChuster, R.M. \& ENGEL, J.J., 1985: Austral Hepaticae, Vol.2. Temperate and subantarctic Schistochilaceae of Australasia. J. Hattori Bot. Lab. 58: 255-539.

ScotT, G.A.M., 1985: SOUTHERN AUSTRALIAN LIVERWORTS. Australian Flora and Fauna Series, No.2. Australian Government Publishing Service, Canberra.

ScotT, G.A.M. \& Bradhsaw, J.A., 1986: Australian liverworts (Hepaticae): annotated list of binomials and checklist of published species with bibliography. Brunonia 8(1): 1-171.

Scotr, G.A.M. \& Stone, I.G., 1976: THE MOSSES OF SOUTHERN AUSTRALIA. Academic Press, London.

Streimann, H. \& Curnow, J., 1989: CATALOGUE OF MOSSES OF AUSTRALIA AND ITS EXTERNAL TERRITORIES. Australian Flora and Fauna Series, No. 10. Australian Government Publishing Service, Canberra.

White, F.J. \& James, P.W., 1985: A new guide to microchemical techniques for the identification of lichen substances. Brit. Lich. Soc. Bull. (suppl.) 57: $1-41$.

Williams, P.R. \& Corbetr, E.B., 1976: Port Davey. Tasm. Geol. Atlas I :50 000 Ser. Sheet SK55-7. Geol. Surv. Tasm.

\section{(accepted 4 May 1990)}

G. Kantvilas

Tasmanian Herbarium, GPO Box 252C, Hobart, Tasmania, Australia 7001

\section{S.J. Jarman}

Forestry Commission, GPO Box 207B, Hobart, Tasmania, Australia 7001; formerlỳ Department of Parks, Wildlife and Heritage 


\section{APPENDIX \\ Vascular Species Recorded from Mount Sprent*}

\section{DICOTYLEDONAE}

APIACEAE

Actinotus bellidioides (J.D.Hook.) Benth.

A. moorei Rodway $\dagger$

A. suffocata (J.D.Hook.) Rodway

Dichosciadium ranunculaceum (F.Muell. ex Hook.) Domin *

Diplaspis cordifolia J.D.Hook. $\uparrow$

Oschatzia saxifraga (J.D.Hook.) Walp. $\dagger$

ARALIACEAE

Pseudopanax gunnii (J.D.Hook.) Philipson $\dagger$

ASTERACEAE

Abrotanella forsteroides (J.D.Hook.) Benth. $\dagger$

A. scapigera (F.Muell.) Benth. $\dagger$

Celmisia asteliifolia J.D.Hook.

C. saxifraga (Benth.) W.M.Curtis $\dagger$

Erigeron stellatus (J.D.Hook.) W.M.Curtis $\dagger$

Ewartia meredithiae (F.Muell.) Beauv. $\dagger$

Helichrysum backhousii (J.D.Hook.) F.Muell. ex Benth. $\dagger$

H. milliganii J.D.Hook. $\dagger$

H. pumilum J.D.Hook. $\dagger$

Olearia ledifolia (DC.) Benth. $\dagger$

O. persoonioides (DC.) Benth. $\dagger$

Senecio leptocarpus DC.

S. pectinatus DC. var. ochroleuca F.Muell. $\dagger$

\section{CUNONIACEAE}

Anodopetalum biglandulosum A.Cunn. ex

J.D.Hook. $\dagger$

Bauera rubioides Andrews

DONATIACEAE

Donatia novae-zelandiae J.D.Hook.

DROSERACEAE

Drosera arcturi Hook.

D. binata Labill.

D. peltata Thunb. ssp. auriculata (Backh. ex

Planchon) Conn

D. pygmeaea $\mathrm{DC}$.

ELAEOCARPACEAE

Aristotelia penduncularis (Labill.) J.D.Hook. $\dagger$

EPACRIDACEAE

Archeria comberi Melville

A. hirtella (J.D.Hook.) J.D.Hook. $\dagger$

A. serpyllifolia J.D.Hook. $\dagger$

Cyathodes juniperina (Forst.) Druce

C. sp. nov. (aff. petiolaris) $\dagger$

Dracophyllum milliganii J.D.Hook. $\dagger$

Epacris corymbiflora J.D.Hook. $\dagger$

E. navicularis Jarman $\dagger$

E. serpyllifolia $\mathrm{R} . \mathrm{Br}$.

Leucopogon milliganii (F.Muell.) Rodway $\dagger$

Monotoca submutica (Benth.) Jarman $\dagger$

Pentachondra pumila (Forst. \& Forst. f.) R.Br.

Prionotes cerinthoides (Labill.) R.Br. $\dagger$

Richea curtisiae A.M.Gray †

R. milliganii (J.D.Hook.) F.Muell. †

R. pandanifolia J.D.Hook. $\dagger$
R. scoparia J.D.Hook. †

Sprengelia distichophylla (Rodway) W.M.Curtis †

S. incarnata Smith

S. incarnata Smith (prostrate alpine form)

Trochocarpa cunninghamii (DC.) W.M.Curtis †

T. gunnii (J.D.Hook.) Benth. †

ERICACEAE

Gaultheria hispida R.Br. $\dagger$

ESCALLONIACEAE

Anopterus glandulosus Labill. †

Tetracarpaea tasmanica J.D.Hook. †

EUCRYPHIACEAE

Eucryphia milliganii J.D.Hook. †

FABACEAE

Acacia mucronata Willd. ex H.Wendl.

FAGACEAE

Nothofagus cunninghamii (Hook.) Oerst.

GENTIANACEAE

Gentianella diemensis (Griseb.) J.H.Willis

LENTIBULARIACEAE

Utricularia lateriflora $\mathrm{R} . \mathrm{Br}$.

LOGANIACEAE

Mitrasacme archeri J.D.Hook.

$M$. montana J.D.Hook. ex Benth.

MONIMIACEAE

Atherosperma moschatum Labill.

MYRTACEAE

Baeckea leptocaulis J.D.Hook. †

Eucalyptus vernicosa J.D.Hook. †

Leptospermum glaucescens $\mathrm{S}$.Schauer $\dagger$

L. nitidum J.D.Hook.

Melaleuca squamea Labill.

PITTOSPORACEAE

Billardiera longiflora Labill.

PROTEACEAE

Agastachys odorata R.Br. $\dagger$

Banksia marginata Cav.

Cenarrhenes nitida Labill. $\dagger$

Orites revoluta $\mathrm{R} . \mathrm{Br}$. $\dagger$

Persoonia gunnii J.D.Hook. †

RANUNCULACEAE

Anemone crassifolia Hook. †

ROSACEAE

Geum talbotianum W.M.Curtis $\uparrow$

RUBIACEAE

Coprosma nitida J.D.Hook.

RUTACEAE

Boronia pilosa Labill.

Phebalium squameum (Labill.) Engler

SANTALACEAE

Exocarpos humifusus R.Br. $\dagger$

Leptomeria glomerata F.Muell. $\dagger$

SCROPHULARIACEAE

Euphrasia hookeri Wettst. $\dagger$

E. sp.

Ourisia integrifolia R.Br. $†$ 


\section{STYLIDIACEAE}

Forstera bellidifolia J.D.Hook. $\dagger$

Stylidiun graminifolium Swartz

THYMELAEACEAE

Pimele lindleyana Meissner $\uparrow$

WINTERACEAE

Tasmannia lanceolata (Poir.) A.C.Smith

\section{MONOCOTYLEDONAE}

CENTROLEPIDACEAE

Centrolepis monogyna (J.D.Hook.) Benth. $\ddagger$

Gaimardia amblyphylla W.M.Curtis $\dagger$

G. fitzgeraldii F.Muell. \& Rodway $\dagger$

\section{CYPERACEAE}

Carphaalpina R.Br.

C. curvata W.M.Curtis $\dagger$

C. rodwayi W.M.Curtis †

Gahnia grandis (Labill.) S.T.Blake

Gymnoschoenus sphaerocephalus (R.Br.) J.D.Hook.

Lepidosperma inops F.Muell. $\dagger$

Oreobolus acutifolius S.T.Blake $\dagger$

O. oligocephalus W.M.Curtis $\dagger$

O. pumilio R.Br.

Schoenus tenuissimus Benth.

Uncinia compacta R.Br.

HYPOXIDACEAE

Campynema lineare Labill. $\dagger$

IRIDACEAE

Diplarrena latifolia Benth. $\dagger$

Isophysis tasmanica (Hook.) T.Moore $\uparrow$

JUNCACEAE

Luzula sp.

LILIACEAE

Astelia alpina R.Br.

Blandfordia punicea (Labill.) Sweet †

Milligania densiflora J.D.Hook. $\dagger$

M. stylosa (F.Muell. ex J.D.Hook.) F.Muell. ex Benth.

ORCHIDACEAE

Acianthus viridis J.D.Hook.

POACEAE

Danthonia fortunae-hibernae Renvoize $\dagger$

D. pauciflora R.Br.
Deyeuxia monticola (Roem. \& Schult.) Vickery

Ehrharta oreophila (D.Morris) Willemse $\dagger$

E. tasmanica (J.D.Hook.) Willemse †

Hierochloe fraseri J.D.Hook. ex Rodway $\dagger$

Poa gunnii Vickery $\dagger$

RESTIONACEAE

Calorophus sp.nov. $\dagger$

Empodisma minus (J.D.Hook.) L.Johnson \& Cutler

Lepyrodia tasmanica J.D.Hook.

Restio complanatus $\mathrm{R}$. Br.

R. monocephalus R.Br. $\dagger$

XYRIDACEAE

Xyris marginata Rendle †

\section{GYMNOSPERMAE}

PHYLLOCLADACEAE

Phyllocladus aspleniifolius (Labill.) J.D.Hook. $\dagger$

PODOCARPACEAE

Podocarpus lawrencii J.D.Hook.

\section{PTERIDOPHYTA}

ASPIDIACEAE

Polystichum proliferum (R.Br.) C.PresI

BLECHNACEAE

Blechnum wattsii Tind.

DENNSTAEDTIACEAE

Histiopteris incisa (Thunb.) J.Smith

GLEICHENIACEAE

Gleichenia dicarpa R.Br.

GRAMMITIDACEAE

Grammitis billardier $i$ Willd.

HYMENOPHYLLACEAE

Apteropteris applanata A.M.Gray \& R.G. Williams Hymenophyllum marginatum J.D.Hook. \& Grev.

H. peltatum (Poir.) Desv.

H. rarum R.Br.

LINDSAEACEAE

Lindsaea linearis $\mathrm{S}$ wartz

LYCOPODIACEAE

Lycopodium fastigiatum $\mathrm{R} . \mathrm{Br}$.

L. laterale $\mathrm{R} . \mathrm{Br}$.

L. varium $\mathrm{R} . \mathrm{Br}$.

* Nomenclature after Buchanan et al. (1989).

$\dagger=$ endemic. 